\title{
TOWARD TEACHING AGENT-BASED SIMULATION
}

\author{
Charles M. Macal \\ Michael J. North \\ Argonne National Laboratory \\ Center for Complex Adaptive Agent Systems Simulation $\left(\mathrm{CAS}^{2}\right)$ \\ 9700 S. Cass Ave. \\ Argonne, IL 60439, USA
}

\begin{abstract}
Agent-based simulation (ABS) is a relatively recent modeling technique that is being widely used to model complex adaptive systems by many disciplines. Few full length courses exist on agent-based modeling and a standard curriculum has not yet been established, but there is considerable demand to include ABS into simulation courses. Modelers often come to agent-based simulation by way of self-study or attendance at tutorials and short courses. Although there is substantial overlap, there are many aspects of ABS that differ from discrete-event simulation (DES) and System Dynamics (SD), including applicable problem domains, disciplines and backgrounds of students, and the underpinnings of its computational implementation. These factors make ABS difficult to include as an incremental add-on to existing simulation courses. This paper reports on some approaches to teaching the modeling of complex systems and agent-based simulation that the authors have used in a range of classes and workshops.
\end{abstract}

\section{INTRODUCTION}

Agent-based simulation (ABS) is a relatively recent modeling technique that is being widely used to model complex systems composed of interacting, autonomous "agents" (Macal and North 2010). Agents have behaviors, which are often described by simple rules. Agents interact with and influence each other, learn from their experiences, and adapt their behaviors so they are better suited to their environment (Figure 1). By modeling agents individually, the full effects of the diversity that exists among agents with respect to their attributes and behaviors can be observed as they give rise to the dynamic behavior of the system as a whole.

A recent sampling of agent-based modeling found applications published in many disciplines ranging from archaeology, biology, and ecology to supply chains, consumer market analysis, military planning, and economics (Macal and North 2009). With this enormous range of applicability, there is substantial interest in and a steadily growing demand for systematic instruction on how to develop and apply agentbased models.

Few full length courses exist on agent-based modeling and a standard curriculum has not yet been established. A standard text does not exist. Modelers often come to agent-based simulation by way of selfstudy or attendance at tutorials and short courses. Agent-based simulation is an alternative simulation approach to System Dynamics (SD) and Discrete-Event Simulation (DES) for modeling systems. There are relationships between ABS and SD or DES, but the full extents of these relationships are not fully understood at this time. There is considerable interest in including ABS into existing simulation courses, whether the courses are focused on DES, SD, or simulation modeling in general. Although there is substantial overlap, many aspects of ABS differ from DES and SD. These include applicable problem do- 


\section{Macal and North}

mains, the disciplines and backgrounds of students, and the underpinnings of the computational implementation. Students come to ABS from many backgrounds and have a wide range of quantitative, computer programming, and modeling backgrounds and learned skills, as well as a range of natural skills and aptitudes for modeling. These factors make ABS difficult to include as an incremental add-on to existing simulation courses.

This paper reports on some approaches to teaching the modeling of complex systems and agent-based simulation that the authors have used in several classes and workshops. In Section 2 we discuss considerations and issues in developing a teaching strategy for ABS. In Section 3 we propose curriculum for such a course that is adaptable to various course lengths and technical proficiencies of the students. In Section 4 we state our conclusions.

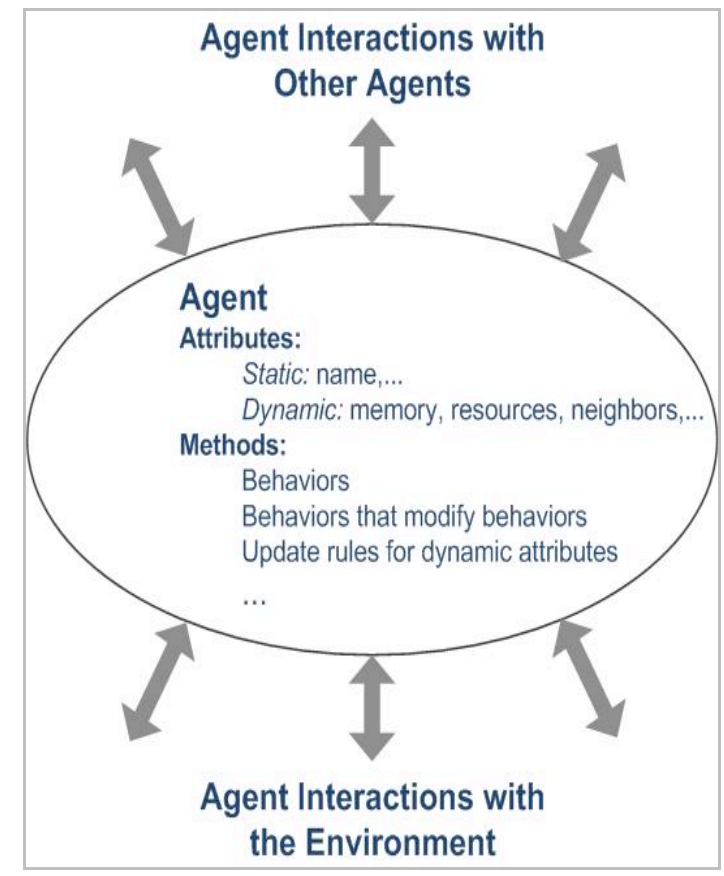

Figure 1: A typical agent. Agents have behaviors and interact with other agents and the environment.

\section{TEACHING AGENT-BASED MODELING AND SIMULATION}

\subsection{The Agent Perspective}

Agent-based modeling offers the agent perspective as its central concept and is a natural starting point for teaching modeling. The agent perspective allows one to come at the modeling problem from the standpoint of the individuals who comprise the system and consider their individual decision-making behaviors and rules. Agent-based modeling allows us to work with models of real, or supposed, agent behaviors, rather than idealized versions and to see what the logical implications are of agent interactions on a large scale. Fewer assumptions have to be made in terms of aggregating agent behaviors or working with only "representative" agents. Operational science has traditionally focused on the process view of the world whereby the process or activity is the central focus of analysis and modeling. The agent-based view of the world is not the traditional approach taken by operational science with its emphasis on process and normative decision making. This in turn has implications for teaching ABS. In effect, we are all agents. And this fact makes teaching agent-based modeling, if done effectively, engaging for both the students and the instructors. 


\section{Macal and North}

\subsection{Pathways to Agents}

Agent-based simulation as a modeling approach, and also agent-based simulation software, did not arise out of the traditional modeling and simulation or operations research fields. Agent-based modeling and simulation can be traced to investigations into complex systems (Weisbuch 1991), complex adaptive systems (Holland 1995, Kaufmann 1993), the evolution of cooperation (Axelrod 1984), and artificial life (Langton 1989) or ALife. (See Macal (2009) for a review of the influences of investigations into artificial life on the development of agent-based modeling, and Heath and Hill (2010) for a review of other early influences.)

Owing to the diversity of its origins and its broad range of applicability, agent-based modeling courses seem to attract highly diverse groups of students. For example, it is quite common in a single short course or tutorial to find professors and students from a range of academic departments such as political science, psychology, sociology, computer science, electrical engineering, environmental science, and even philosophy alongside practicing engineers and business people. The diverse audiences have diverse backgrounds and experiences and skill levels. This naturally leads to a diverse set of prerequisites. Addressing this diversity means insuring that all of the major topics considered below are covered at least at a basic level while still keeping students engaged that are more knowledgeable about each given topic. The backgrounds of the students in large measure determine how agent-based modeling is taught. For example, one way to teach ABS is through the object-oriented (OO) modeling paradigm. If the OO approach is taken to teach $\mathrm{ABS}$, a background in OO design and programming would then be a prerequisite for instruction on ABS.

Agent-based modeling itself demands a diverse skill set. It is rare for one student to possess all of the needed capabilities. What is typically found in practice is that most students have at least basic skills in a few, but not all, of the areas described in Section 3.4. These skills were usually obtained through experiences with different but related activities such as other kinds of modeling, although sometimes the students have worked directly at some level with agent-based models.

\subsection{Introductory Versus Large-Scale ABS}

There are at least two kinds of courses that one could present on agent-based modeling:

- Modeling Complex Systems. An introductory course that emphasizes how to do modeling as much as it does how to do agent-based modeling.

- Building Complex System Models. A course that focuses exclusively on how to use a particular agent-based modeling software toolkit. Within this type of course, there are at least two possibilities:

- Basic Proficiency. A course that focuses on developing a basic proficiency in using a particular agent-based modeling software toolkit.

- Large-scale ABS. One can go beyond basic proficiency in the software and explore advanced applications using the full capabilities and connectivity of the software that would allow one to build large-scale agent-based models.

In an introductory course, one can optionally provide hands-on experience with agent-based modeling software and perhaps build a simple model. Students can be expected to be able to create and build their own agent-based models after such an introductory course that includes hands-on instruction. Large-scale ABS is intended to increase the capabilities of students to develop agent models along one or more of several dimensions including the following:

- $\quad$ Scaling the number of agents upward;

- Increasing the behavioral complexity and memory of agents;

- Increasing the detail level of the simulated agent environment; 


\section{Macal and North}

- Embedding the model into existing organizational work processes, and

- Allowing more developers or users to work on or execute the model.

There are a variety of important tools that can be used to assist with these goals including distributed computing technologies; artificial intelligence and machine learning techniques such as neural networks; geographical information systems (GIS); database systems connections as well as GIS again; version control systems and advanced integrated development environments. Each of these areas could be a course in and of itself, although the knowledge to use these techniques within an agent-based model could be effectively covered as part of an advanced agent based modeling and simulation (ABMS) course.

\subsection{The Agent-based Modeling Conundrum}

The agent-based modeling conundrum refers to the fact that many people come to learn about agent-based modeling and simulation with the serious intent of building models, but without any previous of knowledge or experience with modeling - how to go about modeling, where to begin the process, what models mean, or even without a clear idea of the problem they are trying to solve or the questions they are trying to answer (usually covered in model requirements). This is a side-effect of the mass appeal of agent-based modeling. Yet from the teaching standpoint, one would like to bring agent-based modeling to the masses - to all who are interested in pursuing it. This presents one of the main challenges of teaching agent-based modeling.

\section{PROPOSED AGENT-BASED MODELING COURSE CURRICULUM}

\subsection{Background on Teaching ABMS}

We have taught agent-based modeling at a variety of levels including college courses, academic enrichment seminars, professional education short courses, conference tutorials, and special events. We have also developed a course on agent-based modeling Capturing Business Complexity with Agent-Based Modeling and Simulation that is offered at Argonne on an annual basis in conjunction with the Santa Fe Institute (ANL 2010). The course has been well-attended by a variety of participants over the past eight years. The intention of the course is to impart three things to the students:

- How to think about agent-based modeling and simulation

- How to do agent-based modeling and simulation

- Give the students a language for agent-based modeling and simulation

This course was the basis for the book that we wrote on agent-based modeling and simulation Managing Business Complexity: Discovering Strategic Solutions with Agent-Based Modeling and Simulation (North and Macal 2007), based on our course experiences and as an aid in teaching agent-based modeling and simulation. The book reflects the subjects that have resonated with the students over the first three years the course was taught. The book was written with the intent of being essentially non-technical in the sense that the mathematical background requirements are minimal. This was appropriate given the widely varying background of the students who attend the course.

One of the authors (Macal) has taught graduate-level courses at the University of Chicago on applications of complexity to emergency management; agent-based modeling plays a central role in this course in teaching systems concepts, model design, and management principals. One of the authors (North) has taught separate college courses in three different academic departments at the Illinois Institute of Technology (Computer Science, Design, and Social Science) and academic enrichment seminars including two sessions of the Santa Fe Institute Complex Systems Summer School. We have also taught professional education short courses, tutorials at many conferences including five years at the Winter Simulation Conference, five years at the INFORMS Annual Meeting, and five years at the Military Operations Research 


\section{Macal and North}

Society Symposium, and invited talks at many special events including tutorials for business and government. Course lengths vary from one hour to an entire semester. During these experiences, we have made a variety of observations and solicited feedback from course participants. We have made adjustments to the course curriculum on a continuing basis in response.

\subsection{General Course Outline}

Given the diversity of backgrounds of course participants interested in learning agent-based modeling, we have taken a comprehensive approach in teaching ABS to include instruction on how to do modeling in general. This is much more comprehensive than would be necessary if one focused only on teaching how to use a specific piece of software, such as an agent-based modeling toolkit. But this comprehensive approach is necessary because the need is there.

The following general outline provides a skeleton for a general course on agent-based modeling:

1. Introduce the basic concepts of agent-based modeling;

2. Discuss when and why agent-based models are used;

3. Detail the agent-based model design process;

4. Present a comparative survey of modeling methodologies;

5. Describe a range of tools for agent-based modeling;

6. Discuss one or two specific selected agent-based model development tools;

7. Describe basic agent-based model architectures;

8. Present model verification and validation techniques;

9. Introduce data collection and cleaning for modeling;

10. Cover model output analysis;

11. Describe approaches to presenting results to decision makers; and

12. Detail agent-based model project management.

Topics 8-12 above have much in common with what would be taught in a general class on modeling and simulation as covered, for example, in the textbook by Law (2007). Model verification seeks to insure that model designs are correctly bound into executable code. Model validation seeks to insure that the design and resulting executable code both conform to the topics of interest relative to the underlying questions. Data collection and cleaning skills are used to identify, obtain, test, and configure inputs for models. These skills are essential due to the large fractions of practical project time often spent on data collection and cleaning. Model analysis skills allow model users to setup, execute and then investigate scenarios of interest. This is again relative to the underlying questions. Model results communication skills allow model users to relate their analysis findings, as well as describe agent-based modeling and the relevant details of their particular model, to appropriate audiences. This involves both general communications skills and knowledge specific to agent-based modeling. Project management allows model creators and users to translate goals into results. As with model results communication skills, agent-based modeling project management involves both general knowledge and specific applications for agent-based modeling. Each of these general modeling topics is supplemented with instruction on how it is related to agent-based modeling.

\subsection{Demonstrations}

In-class demonstrations of simple agent-based simulations prove very useful in teaching ABMS concepts and capturing the attention of students. Demonstrations also give students motivation and targets for developing their own initial simulations. The following demonstrations illustrate key points in understanding and motivating ABS:

- Conway's Game of Life - illustrates how simple rules can lead to complex system behaviors 


\section{Macal and North}

- Boids Flocking Simulation - illustrates emergence of order arising out of social interaction

- Schelling Housing Segregation Model - illustrates the use of agent-based modeling to address a social phenomenon

- Mass Opinion Spreading (Information Infectivity) Simulation - illustrates non-linearity, tipping points, and extreme sensitivity to initial conditions

- "Matching Triangles" Participatory Simulation - illustrates how computers can actually simulate how people interact, over a limited knowledge domain

- The Beer Game Participatory Simulation (also known as the Supply Distribution Game) - illustrates how difficult it is to manage complex systems

Before each demonstration, the system being modeled, including the rules of behavior used for the agents is described to the students. Students are asked to anticipate the system behavior they will observe in the demonstration. Afterwards, students discuss what they observed and characterize the results of the demonstrated models in terms of the systems principles discussed in class.

\subsection{Skills}

A participant has who completed the course should have familiarity with and some degree of skill in each area:

- Modeling

- Programming

- Model verification and validation

- Data collection and cleaning

- Model analysis

- Model results communication

- Project management

These general skills follow the outline provided by the Project Management Institute (PMI 2008). The depth at which each of the skills listed is taught depends on the audience and the amount of time available. Students with higher skill levels and longer classes can allow skills to be developed in greater depth. The list of basic skills also determines the prerequisites. In this case, the requirements to take the class are anything on the list that will not be taught during the class.

When considering the skill list, it is important to note that modeling is not programming! There are relationships between these skills, but they are distinct. Simply put, modeling creates abstract representations of topics of interest relative to well-defined questions. Programming binds abstract designs into computer executable code and associated supporting data. The abstract representations created by modeling are one type of design with the potential to be programmed. Given these definitions, it has been our experience that there are successful modelers who do not know how to program, and there are productive programmers who are not adept at, or interested in, modeling. Of course, some people can both model and program.

\subsection{Measuring Student Achievement}

Given the objectives discussed above, how can achievement be measured? Upon successful completion of an introductory course the students should be able to:

1. Define the agent-based modeling process;

2. Compare and contrast agent-based modeling with alterative and complementary techniques and articulate their relative strengths and weaknesses. Other techniques relevant to agent-based mod- 


\section{Macal and North}

eling include system dynamics modeling, discrete-event simulation, participatory simulation, optimization modeling, game theory, statistical modeling, and risk analysis;

3. Describe when and why individuals and organizations use agent-based modeling and simulation;

4. Describe how to design agent-based simulations.

5. Describe how to develop agent-based simulations;

6. Detail how to manage agent-based modeling projects;

7. Demonstrate how to develop models using one or two agent-based model development tools;

8. Articulate how to perform basic model verification and validation;

9. Detail the primary challenges and techniques of data collection and cleaning for modeling;

10. Discuss how to perform basic model output analysis; and

11. Describe how to present model results to decision makers.

\subsection{Course Agendas}

A sample agenda for a week long course on agent-based modeling is shown in Table 1:

Table 1: Outline for a week-long introductory course on agent-based modeling of complex systems

\begin{tabular}{|c|c|c|c|}
\hline Day & Start Time & End Time & Topic \\
\hline \multirow[t]{8}{*}{1} & 9:00 AM & $10: 45$ AM & Introduction to ABMS \\
\hline & 10:45 AM & 11:00 AM & Break \\
\hline & 11:00 AM & 12:00 Noon & When and Why to Use ABMS \\
\hline & 12:00 Noon & 1:00 PM & $\begin{array}{l}\text { Lunch (Ask the Participants to Think of Topics They } \\
\text { May Want to Model with ABMS) }\end{array}$ \\
\hline & 1:00 PM & 2:00 PM & Class Discussion of Participant Suggested Topics \\
\hline & 2:00 PM & 3:00 PM & Comparative Survey of Modeling Methodologies \\
\hline & 3:00 PM & $3: 15 \mathrm{PM}$ & Break \\
\hline & 3:15 PM & 5:00 PM & Agent-based Model Design \\
\hline \multirow[t]{8}{*}{2} & 9:00 AM & 10:30 AM & Tools for ABMS \\
\hline & 10:30 AM & $10: 45 \mathrm{AM}$ & Break \\
\hline & 10:45 AM & 12:00 Noon & Agent-based Model Architectures \\
\hline & 12:00 Noon & 1:00 PM & $\begin{array}{l}\text { Lunch (Ask the Participants to Think of Topics They } \\
\text { May Want to Model with ABMS) }\end{array}$ \\
\hline & 1:00 PM & 2:00 PM & Class Discussion of Participant Suggested Topics \\
\hline & 2:00 PM & 3:15 PM & Verification and Validation \\
\hline & 3:15 PM & 3:30 PM & Break \\
\hline & $3: 30 \mathrm{PM}$ & 5:00 PM & Data Collection and Cleaning \\
\hline \multirow[t]{6}{*}{3} & 9:00 AM & 10:00 AM & Model Output Analysis \\
\hline & 10:00 AM & 11:00 AM & Results Presentation \\
\hline & 11:00 AM & 11:15 AM & Break \\
\hline & 11:15 AM & 12:00 Noon & ABMS Project Management \\
\hline & 12:00 Noon & 1:00 PM & Break \\
\hline & 1:00 PM & 5:00 PM & \multirow{3}{*}{$\begin{array}{l}\text { Selected ABMS Tool Hands-on Tutorial (The audi- } \\
\text { ence writes the model following the instructor step- } \\
\text { by-step) (Include breaks and lunches as appropriate) }\end{array}$} \\
\hline 4 & 9:00 AM & 5:00 PM & \\
\hline 5 & 9:00 AM & 5:00 PM & \\
\hline
\end{tabular}

This week-long course can be scaled down to three-day and one-day versions. Especially in the oneday course, students come away with a deeper understanding of agent-based modeling and information on finding resources from which they can learn more. 


\section{Macal and North}

\subsection{Quarter or Semester Course Outline}

A sample agenda for a quarter or semester course on agent-based modeling is shown in Table 2:

Table 2: Outline for a quarter or semester course on agent-based modeling of complex systems

\begin{tabular}{|c|c|c|}
\hline $\begin{array}{c}\text { Class } \\
\text { Session }\end{array}$ & Topic & Assignments \\
\hline 1 & $\begin{array}{l}\text { Introduction to ABMS } \\
\text { When and Why to Use ABMS } \\
\text { Project Introduced }\end{array}$ & Decide on an ABMS Project Topics \\
\hline 2 & $\begin{array}{l}\text { Comparative Survey of Modeling } \\
\text { Methodologies }\end{array}$ & $\begin{array}{l}\text { Consider ABMS vs. Other Options for the } \\
\text { Selected Project }\end{array}$ \\
\hline 3 & Agent-based Model Design & $\begin{array}{l}\text { Develop a Basic Design for the Selected Pro- } \\
\text { ject }\end{array}$ \\
\hline 4 & Tools for ABMS & $\begin{array}{l}\text { Consider Which ABMS Tool Might be Best } \\
\text { for the Selected Project }\end{array}$ \\
\hline $\begin{array}{c}5,6, \text { and } \\
7\end{array}$ & $\begin{array}{l}\text { Selected ABMS Tool Hands-on Tu- } \\
\text { torial }\end{array}$ & $\begin{array}{l}\text { Implement a Simple Model Assigned by the } \\
\text { Instructor or Extend an Existing Model (See } \\
\text { Zombie Discussion Below) }\end{array}$ \\
\hline 8 & Midterm Exam & Begin Implementing the Selected Project \\
\hline 9 & Agent-based Model Architectures & $\begin{array}{l}\text { Consider Which Architecture Might be Best } \\
\text { for the Selected Project }\end{array}$ \\
\hline 10 & Verification and Validation & $\begin{array}{l}\text { Define How Verification and Validation } \\
\text { Might be Done for the Selected Project }\end{array}$ \\
\hline 11 & Data Collection and Cleaning & $\begin{array}{l}\text { Find and Configure At Least One Data } \\
\text { Source for the Selected Project }\end{array}$ \\
\hline 12 & $\begin{array}{l}\text { Model Output Analysis } \\
\text { Results Presentation }\end{array}$ & $\begin{array}{l}\text { Execute and Analyze the Selected Project's } \\
\text { Model }\end{array}$ \\
\hline 13 & ABMS Project Management & Prepare for the Project Presentation \\
\hline 14 & Project Presentations & \\
\hline 15 & Final Exam & \\
\hline
\end{tabular}

The quarter and semester formats are differentiated by the level of detail covered for each topic. Projects can be completed individually by the students or in small groups.

North's favorite assignment for initial users of an agent modeling platform is to ask the students to add 'zombie' agents (a la George Romero) to any model from the chosen toolkit's example or demonstration models library. The idea of zombies seems to interest most students and is usually easy to explain without technical details. Furthermore, the explanation of the problem can usually be provided without divulging the solution strategy. Determining the solution strategy is left to the students as a learning exercise.

For the reader, it turns out to be relatively simple to add zombies to many agent-based models. The solution strategy is to add a new state variable to existing agents that marks them as either 'living' or 'undead.' Undead (e.g., zombie) agents seek out and bite living agents. Bitten living agents become zombies after a short incubation delay which can be implemented as a simple counter or as a scheduled event, depending on the modeling environment. The zombie's hunting and biting behaviors are programmed by adding simple conditional statements that override the agent's normal behavior and activate the zombie behavior when the agent's state is set to 'undead.' As previously stated, this conceptual design is not provided to students as most are able to figure it out for themselves. 


\section{Macal and North}

Nearly all students seem to be able to complete the 'Zombies!' assignment and a high level of excitement is usually reported. Asking the students to briefly present their extended models to the class during the session that the assignment is due seems to further increase student engagement.

\section{SUMMARY AND CONCLUSIONS}

We have taught agent-based modeling and simulation in a variety of venues over the past nine years. These include a week-long course offered at Argonne, tutorials at professional conferences, invited presentations before industrial trade groups and private companies, and academic courses at the University of Chicago (Macal) and the Illinois Institute of Technology (North). We have arrived at the curriculum presented here that focuses on learning how to think about and how to do agent-based modeling. The curriculum also includes many aspects of modeling and simulation in general, out of necessity due to the diverse backgrounds and interests of course participants. The curriculum has met with success in terms of reported student satisfaction; growth in course interest and attendance over the years; testable student capabilities to develop models on their own; and documented examples of students independently developing models following their training. Yet, there is room for improvement, and much work needs to be done in the future on understanding the most effective ways to teach agent-based modeling and simulation.

\section{ACKNOWLEDGMENTS}

This work was supported by the U.S. Department of Energy under contract number DE-AC02$06 \mathrm{CH} 11357$.

\section{REFERENCES}

ANL 2010. Capturing Business Complexity with Agent-Based Modeling and Simulation: Useful, Usable, and Used Techniques. Argonne National Laboratory. Available via <http://www.dis.anl.gov/conferences/abms/info.html>[accessed April 14, 2010].

Axelrod, R. 1984. The evolution of cooperation. Perseus Books.

Heath, B. L., and R.R. Hill. 2010. Some insights into the emergence of agent-based modeling. Journal of Simulation. in press.

Holland, J. 1995. Hidden order: how adaptation builds complexity. Reading, MA: Addison-Wesley.

Kauffman, S. A. 1993. The origins of order: self-organization and selection in evolution. Oxford UK: Oxford University Press.

Langton, C. G. 1989. Artificial life, in Artificial Life: The Proceedings of an Interdisciplinary Workshop on the Synthesis and Simulation of Living Systems, Held September 1987, Los Alamos, New Mexico (Volume VI in Santa Fe Institute Studies in the Sciences of Complexity) Langton, C. G. (ed.) pp. 147. Reading, MA: Addison-Wesley.

Law, A. M. 2007. Simulation modeling and analysis. 4th ed. McGraw-Hill.

Macal, C. M. 2009. Agent based modeling and artificial life. In Encyclopedia of Complexity and Systems Science, ed. R. Meyers, 112-131. Springer.

Macal, C., and M. J. North. 2009. Agent-based modeling and simulation. In Proceedings of the 2009 Winter Simulation Conference, ed. M. D. Rossetti, R. R. Hill, B. Johansson, A. Dunkin, and R. G. Ingalls, 86-98. Piscataway, New Jersey: Institute of Electrical and Electronic Engineers, Inc.

Macal, C., and M. J. North. 2010. Tutorial on agent-based modeling and simulation. Journal of Simulation, in press.

North, M. J., and C. M. Macal. 2007. Managing business complexity: discovering strategic solutions with agent-based modeling and simulation. Oxford, U.K.: Oxford University Press.

PMI 2008. A Guide to the Project Management Body of Knowledge, $4^{\text {th }}$ Ed. Project Management Institute. 
Weisbuch, G. 1991. Complex systems dynamics: an introduction to automata networks (Translated from French by S. Ryckebusch). Redwood City, CA: Addison-Wesley.

\section{AUTHOR BIOGRAPHIES}

CHARLES M. MACAL, Ph.D., P.E., is the Director of the Center for Complex Adaptive Agent Systems Simulation, Argonne National Laboratory, Senior Fellow in the joint Computation Institute of the University of Chicago and Argonne, and Adjunct Professor in the Graham School of the University of Chicago. $\mathrm{He}$ is a member of the INFORMS Simulation Society, the Association for Computing Machinery, the Society for Computer Simulation International, the Systems Dynamics Society and a founding member of the Computational Social Simulation Society. Dr. Macal has a Ph.D. in Industrial Engineering \& Management Sciences (Operations Research) from Northwestern University and a Master's Degree in Industrial Engineering (Computer Simulation) from Purdue. He is also a Registered Professional Engineer. His email address is $\langle$ macaleanl.gov $>$.

MICHAEL J. NORTH, M.B.A., Ph.D., is the Deputy Director of the Center for Complex Adaptive Agent Systems Simulation within the Decision and Information Sciences Division of Argonne National Laboratory, a Senior Fellow in the joint Computation Institute of the University of Chicago and Argonne, and an Adjunct Professor of Design in the Institute of Design at the Illinois Institute of Technology. Dr. North has over fifteen years of experience developing and applying advanced modeling and simulation applications for various branches of the U.S. federal government; state government; several international agencies; private industry; and academia. Dr. North is the lead author of the book Managing Business Complexity: Discovering Strategic Solutions with Agent-Based Modeling and Simulation (Oxford 2007) and has authored or co-authored over fifty journal articles and conference papers. His email address is 